\title{
Effects of Free Surface and Heterogeneous Residual Internal Stress on Stress-Driven Grain Growth in Nanocrystalline Metals
}

\author{
F. Wang, ${ }^{1}$ J. Zhao, ${ }^{1}$ P. Huang, ${ }^{2}$ A. S. Schneider, ${ }^{3}$ T. J. Lu, ${ }^{1}$ and K. W. Xu ${ }^{2}$ \\ ${ }^{1}$ State Key Laboratory for Strength and Vibration of Mechanical Structures, School of Aerospace, Xian Jiaotong University, \\ Xian 710049, China \\ ${ }^{2}$ State-Key Laboratory for Mechanical Behavior of Material, Xi'an Jiaotong University, Xian 710049, China \\ ${ }^{3}$ INM-Leibniz Institute for New Materials, Campus D2 2, 66123 Saarbrucken, Germany
}

Correspondence should be addressed to F. Wang; wangfei@mail.xjtu.edu.cn and P. Huang; huangping@mail.xjtu.edu.cn

Received 18 June 2013; Revised 24 August 2013; Accepted 28 August 2013

Academic Editor: Tianxi Liu

Copyright (C) 2013 F. Wang et al. This is an open access article distributed under the Creative Commons Attribution License, which permits unrestricted use, distribution, and reproduction in any medium, provided the original work is properly cited.

By reevaluating the experimental study of Zhang et al. (2005), here we demonstrate that the extent of grain growth, previously proposed to be solely driven by external stress, may have been significantly overestimated. A new physical mechanism, termed as free surface assisted stress-driven grain growth (or self-mechanical annealing), is proposed and discussed in detail. Representing the cooperative effect of free surface and heterogeneous residual internal stress, the proposed mechanism is considered more favorable than the traditional pure stress-driven mechanism for interpreting the abnormal grain growth widely observed in deforming nanocrystalline metals at room temperature.

Whilst grain boundaries (GBs) in polycrystalline metals are traditionally described as a mechanically static and immovable microstructural obstacle to dislocation motion, numerous experimental studies have provided convincing evidence that GBs are not as static as traditionally assumed in nanocrystalline (NC) metals [1-6]. These studies indicated further that the unusual mobility of GBs under applied stress may eventually lead to rapid grain growth in NC metals. Also, surprisingly, the grain growth was found to be faster at cryogenic temperature than at room temperature (RT), suggesting that the grain coarsening process was driven primarily by stress but not diffusion $[3,5,6]$. As the classical models for grain growth failed to explain the observed grain structure instability in $\mathrm{NC}$ metals, identifying the underlying mechanism of the unique grain growth process has become a hot research topic.

Before clarifying the underlying mechanism, however, existing inconsistency concerning the mechanical properties of NC metals needs to be addressed in advance. Contrary to prevalent experimental reports of grain growth in NC metals $[1,2,4-9]$ by in situ or post mortem transmission electron microscopy (TEM) analysis, bulk NC metals exhibiting remarkable grain structure stability have also been reported $[10,11]$. For typical instance, whilst significant stress-assisted grain growth was observed in an $800 \mathrm{~nm}$ grained Al thin film by means of in situ TEM straining experiments [2], however, it has never been observed in bulk nanostructured face-centered cubic (FCC) metals having similar grain size. As grain growth is commonly observed by TEM observation, this inconsistency may arise from surface effects inherent to TEM experiments. Given that the thin foils are less than a few micrometers in thickness, it remains unclear whether the observed GB and dislocation structures are representative of material structure during deformation, or just artifacts from thinning [12]. For in situ testing of ultrathin TEM foils, the remarkable grain instability may be enhanced by significant diffusion from nearby free surfaces, leading possible wrong interpretations of the coarsening extent. Ex situ TEM observations, on the other hand, may be influenced by stress relaxation during specimen preparation (e.g., sample thinning to submicron scale). Accordingly, identifying the dominating mechanism of stress-driven grain growth of $\mathrm{NC}$ metals at RT in NC metals and predicting quantitatively how fast and how far the stress-driven grain growth could 


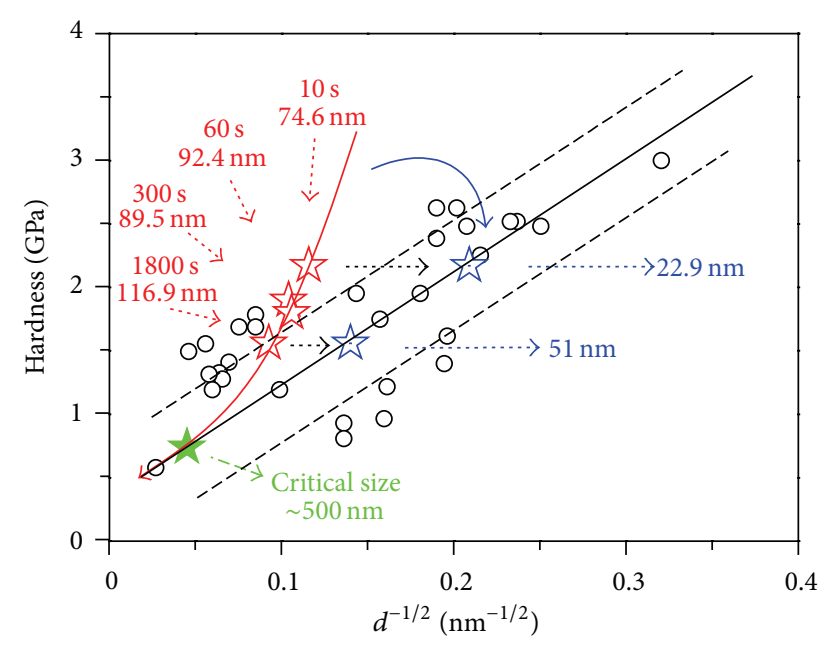

O Chen et al.

¿ Zhang et al.

通 Fit to H-P relation

FIgURE 1: Grain size dependent hardness of $\mathrm{Cu}$. The circles and the generated trend line of Hall-Petch relation are taken from the work of Chen et al. [13], and the red stars are data reproduced from the work of Zhang et al. [5].

be are of great scientific interest. The aim of this letter is to suggest new physical mechanism governing grain structure instability of NC metals that may significantly increase the extent of grain growth, by considering the cooperative effects of a free surface and heterogeneous residual stresses.

To rationalize the above hypothesis, we begin by reevaluating the work of Zhang et al. [5], which, as a milestone in the research field of stress-driven grain growth, is amongst the most cited studies thus far. In this study, a number of remarkable observations were made by nanoindentation and post mortem TEM analysis, including the following: (1) whilst the indentation hardness continually decreased with time for $\mathrm{NC} \mathrm{Cu}$, it did not change significantly for coarse grain $\mathrm{Cu}$ under the same circumstances; (2) the mechanical coarsening process, which was thought to be responsible for the decreasing hardness, was extremely fast; for example, the grain size increased more than five times within a dwell time of only $10 \mathrm{~s}$ in indentation creep test; (3) grain growth was faster at $-190^{\circ} \mathrm{C}$ than at RT, which provides a crucial evidence that the grains could grow purely under the action of stress without any diffusional processes involved. However, the hardness measured for a specific grain size significantly deviates from other studies as shown in Figure 1. This indicates that the amount of grain growth as reported by Zhang et al. [5] was overestimated by ignoring several key parameters, for example, surface effects, intrinsically involved in preparing TEM samples.

In order to rationalize this assumption, a comparison between the test data presented by Zhang et al. [5] and the Hall-Petch (H-P) relation was made in Figure 1, with circles and the trend lines representing the $\mathrm{H}-\mathrm{P}$ relation of pure $\mathrm{Cu}$ [13]. The results of Figure 1 demonstrate clearly that, based on the grain sizes calculated from Figure 2 in [5] (also see
Figure 13 in [14] for better resolution), the hardness derived by Zhang et al. deviates significantly from the classical $\mathrm{H}$ $\mathrm{P}$ relation for $\mathrm{Cu}$. For example, the grain size of $74.6 \mathrm{~nm}$ observed after $10 \mathrm{~s}$ dwell time is more than three times larger than that obtained by fitting to H-P relation, that is, $22.9 \mathrm{~nm}$ as shown in Figure 1. After $1800 \mathrm{~s}$ dwell time, the grain size of $116.9 \mathrm{~nm}[5,14]$ observed in post modern TEM is still more than twice larger than that fitting to the H-P relation. Note that the hardness (shown as red star in Figure 1) derived from indentation test should reflect the original grain size, that is, before the $\mathrm{Cu}$ sample was processed into a TEM foil. Consequently, the comparison shown in Figure 1 indicates that the grain sizes determined form postmortem TEM are significantly larger than those corresponding to indentation hardness. This observation suggests further that the grain size may only increase $\sim 3 \mathrm{~nm}$ and $\sim 41 \mathrm{~nm}$ after $10 \mathrm{~s}$ and $1800 \mathrm{~s}$ dwell time, respectively, in comparison with the initial average grain size of $20 \mathrm{~nm}$ for inert gas condensation (IGC) $\mathrm{Cu}$ reported by Zhang et al. [5]. Given that the grains directly underneath the indenter should exhibit a gradient distribution along the direction away from the indenter tip and that all the grains involved in the deformed region contribute to the indention hardness, the extent of grain growth must be larger than $3 \mathrm{~nm}$, although this is still significantly overestimated. In other words, the majority of the extent of grain growth (specifically, several tens of nanometers for the test sample with $10 \mathrm{~s}$ dwell time) observed in post mortem TEM may be induced by mechanisms other than pure stressdriven process. In other words, additional mechanisms may exist and play a crucial role in initiating and promoting the grain growth in NC metals.

Upon deformation, yielding should occur first in relatively larger grains, with smaller grains accommodating the deformation elastically. This result in inhomogeneous stress distribution and significant residual internal stress built up in NC metals [15]. Even though the inhomogeneous stress distribution as well as the residual internal stress could be somewhat relaxed during unloading, the majority of the residual internal stress would remain after unloading for the deformed region is restricted by the surrounding undeformed material. In some certain circumstances, the residual internal stress remaining after unloading could affect the plastic deformation of NC metals in an unexpected way as addressed below.

In a study on the plastic deformation in free-standing NC metallic thin films, Rajagopalan et al. found that a substantial fraction of the plastic deformation could be recovered after unloading and subsequent annealing for a $50 \mathrm{~nm}$ grained $\mathrm{Al}$ film but not for $180 \mathrm{~nm}$ grained Au film [16]. This recovery process was proposed to be a thermally activated and time dependent event. Two mechanisms have been proposed to interpret the plastic deformation recovery: thermally activated dislocation jumps over grain boundary obstacles $[16,17]$ and heterogeneous grain boundary diffusion and sliding $[15,18]$. Despite the fact that these two microscopic processes are quite different, both mechanisms considered the heterogeneity of the stress distribution; that is, large internal residual stresses built-up during plastic deformation play a dominant role in the recovery phenomena [15-18]. It 
TABLE 1: Experimental data on deformation induced grain growth in NC metals and alloys.

\begin{tabular}{|c|c|c|c|c|c|}
\hline Material (synthesis) & $d_{\text {initial }}(\mathrm{nm})$ & Deformation mode & $d_{\text {final }}(\mathrm{nm})$ & Mechanism & Reference \\
\hline $\mathrm{Al}$ (MST) & 20 & In situ TEM indentation & $>100$ & GBM, GR, GC & {$[22]$} \\
\hline $\mathrm{Ni}(\mathrm{ED})$ & 45 & Compression creep & 75 & GBS, GR & {$[23]$} \\
\hline $\mathrm{Al}(\mathrm{MST})$ & $40-90$ & Tension & $>100$ & Stress coupled GBM & {$[4]$} \\
\hline Co-P (ED) & 12 & Tension & 25 & Stress-driven GBM, GC & [24] \\
\hline $\mathrm{Ni}-\mathrm{Fe}(\mathrm{ED})$ & 23 & Tension & 250 & Stress-driven GB process & [25] \\
\hline $\mathrm{Ni}(\mathrm{ED})$ & 30 & HPT & 130 & Stress assisted GR & {$[8]$} \\
\hline $\mathrm{Ni}(\mathrm{ED})$ & 15 & Multi-indentation & $<200$ & GB processes & {$[26]$} \\
\hline $\mathrm{Cu}(\mathrm{IGC})$ & 36 & Compression & 90 & Stress-driven mechanism & [27] \\
\hline $\mathrm{Ni}(\mathrm{ED})$ & 15 & In situ TEM tension & $>50$ & GR & {$[28]$} \\
\hline $\mathrm{Ni}(\mathrm{ED})$ & 37 & Rolling & 110 & GB-dislocation interaction & [29] \\
\hline $\mathrm{Ni}(\mathrm{ED})$ & 42 & & & & \\
\hline $\mathrm{Ni}-\mathrm{Fe}(\mathrm{ED})$ & 25 & Fatigue in tension & $>500$ & Diffusional shear stress-driven growth & [30] \\
\hline Ni-Mn (ED) & 115 & & & & \\
\hline $\mathrm{Ni}-\mathrm{Fe}(\mathrm{ED})$ & 20 & Rolling & 50 & GR & {$[31]$} \\
\hline Pt (MST) & 20 & Tension & 33 & Stress-driven GB process & {$[32]$} \\
\hline $\mathrm{Ni}-\mathrm{Fe}(\mathrm{ED})$ & 21 & HPT & 50 & GR & [33] \\
\hline
\end{tabular}

MST: magnetron sputtering technique; IGC: inert gas condensation; ED: electrodeposition; HPT: high pressure torsion; GBM: GB migration; GR: grain rotation; GC: grain coalescence.

is assumed that the heterogeneity of the stress distribution provides the driving force for the reverse plastic deformation, which is then triggered by an annealing treatment at a higher temperature [16]. In a similar way, peeling restricting regions in preparing the TEM sample may trigger the redistribution of the heterogeneous stress remaining after indentation deformation and thus might cause additional grain growth.

Built upon existing studies as discussed above, we hypothesize that the mechanism(s) that corresponds to the additional grain coarsening observed by post mortem TEM is closely related to stress relaxation (i.e., reducing the heterogeneity of stress distribution) during TEM sample preparation, namely, free surface assisted stress-driven grain growth. To this end, two factors are crucial: significant residual internal stresses and free surface effect. Although the precise microstructural processes that can attribute to the additional extent of grain growth are yet clear, numerous potential deformation mechanisms may be activated in the additional coarsening process. As only a few grains are sitting atop each other and due to the proximity of the free surface in the TEM foil, surface effects could enhance both GB- and dislocationmediated deformation processes significantly [19-21]. Table 1 summarizes existing experimental data $[4,8,22-33]$ concerning abnormal grain growth in NC metals and alloys at $300 \mathrm{~K}$ (except those in [23] obtained at $373 \mathrm{~K}$ ); the corresponding synthesizing method, initial grain size $d_{\text {initial }}$, testing method, final grain size $d_{\text {final }}$, and proposed dominant deformation mechanisms are also listed. It can be seen that the majority of the proposed grain coarsening processes involve GB motions under high stress (i.e., the so-called stress-driven or stress assisted GB activities), for example, GB sliding, GB rotation, GB migration, GB diffusion and even dislocation motions, and so forth. Apparently, no matter which one of these candidate mechanisms dominates the coarsening process, enlarging grain size requires a decrease in the volume fraction of GBs; that is, parts of the GBs present after unloading should be eliminated upon the thinning process.

Concerning dislocation movement, Rajagopalan et al. suggested that dislocation propagation in the plastically deformed NC metals would occur upon unloading and is responsible for the time evolution of strain recovery. However, neither molecular dynamics (MD) simulations [34] nor experiments [35] have revealed that dislocation nucleation and propagation induces grain growth in NC metals. Therefore, whilst such dislocation movement may be active in the free surface assisted stress-driven grain growth process, dislocation propagation could hardly move GBs and hence only serve as an accessory deformation mechanism in the coarsening of nanoscale grains. Opposed to that, numerous studies concerning in situ mechanical testing indicated that the applied stress could indeed cause significant GB migration while the dimension of the sample is in submicron scale $[2,36]$. Note that the critical dimension of these sub-micrometer sized test samples is quite similar to those of a typical TEM foil. Thus, it is very likely that the internal stress in a TEM sample can induce grain growth upon the thinning procedure. The only difference is that the driving force changes from the external stress to the relaxation of the high residual internal stress.

The mechanism which causes the grain growth in the subsurface region might involve numerous GB motions, for example, shear-coupled GB migration, GB rotation, GB diffusion and GB sliding, and so forth. Specifically, in NC Al thin films, Rupert et al. [1] showed that GBs could be moved by shear stress in a manner of coupled grain boundary migration. The shear-coupled GB migration, initially modeled in a bicrystal configuration and later confirmed afterward by experimental [1] and simulation [37] studies, 
has been proposed as an effective way for stress relaxation by generating permanent shear [38]. In a similar way, the shearcoupled GB migration could occur in a thinning TEM foil via relaxation of high residual internal stress. On the other hand, Bobylev and Ovid'ko recently proposed a new mechanism for $\mathrm{GB}$ rotation in $\mathrm{NC}$ metals, that is, stress-driven rotations of GBs in subsurface areas of NC solids [21]. Here, the free surface provides an effective sink for GBs in reducing their length. This mechanism may indeed occur in stress-driven grain growth, as evidence of grain rotation dominating the coarsening process was found in NC Cu film by post-TEM observations [39] (it is worth mentioning that the sample preparation and indentation procedures as reported in [39] and [5] were identical). However, this kind of GB rotation can only affect the outmost grains and its contribution to additional grain growth is not as large as shear-coupled GB migration. In addition, heterogeneous grain boundary diffusion and sliding as proposed by Wei et al. $[15,18]$, which are also driven by the heterogeneity of the stress distribution in NC metals with submicron scale thickness, could also play a key role in the additional grain growth.

Taking as a whole, we cannot rule out any of the three possible mechanisms discussed above as the dominant one in the free surface assisted stress-driven grain growth process. To this end, elaborate experiments are clearly needed, as experimentally separating the extent of grain growth between the two physical processes, that is, pure stress-driven grain growth and the free surface assisted stress-driven grain growth, is extremely challenging. Therefore, numerical simulations, for example, MD simulation, may be more suitable for this task.

Other than experimental observation of grain growth in post-TEM indentation (specifically, [5] in this study), it should be mentioned that the mechanism of free surface assisted stress-driven grain growth may occur in all thinning TEM samples which underwent mechanical stress driven grain growth as shown in Table 1 [8, 23-27, 29-31, 33] and hence can shed some light on the interpretation of grain growth in in situ mechanical testing where no thinning process is required after deformation [4, 22, 28, 32]. Moreover, there must be a critical grain size above which the additional extent of grain growth induced by the proposed mechanism is negligible. On the basis of the trend line following Zhang et al.s data [5] shown in Figure 1, we speculate that the critical grain size may be as large as roughly $500 \mathrm{~nm}$.

Further, the mechanism proposed in the present study could explain, to certain extent, why grain growth was faster at $-190^{\circ} \mathrm{C}$ than that at RT in NC Cu. As the higher indentation hardness derived at $-190^{\circ} \mathrm{C}$ corresponds to larger residual internal stress located inside the nanoscale grains, the free surface assisted stress-driven grain growth should be more pronounced than that at RT. Therefore, for samples having identical grain size before deformation, the additional extent of grain growth should be much larger at $-190^{\circ} \mathrm{C}$ than that at RT.

In conclusion, by reevaluating the experimental observations reported by Zhang et al. [5], our analysis reveals that the extent of grain growth driven by pure mechanical stress may be significantly overestimated. A new physical mechanism of grain growth driven by both mechanical stress and free surface effect is proposed for NC metals. Whilst the dominant mechanism(s) is yet to be identified specifically, it is clear that several GB-mediated mechanisms may contribute to the additional grain growth. However, to what extent the free surface assisted stress-driven grain growth is related to the total grain growth observed under TEM remains a challenge.

\section{Acknowledgments}

The present work was supported by the National Basic Research Program of China (2010CB631002, 2011CB610306,), the National Natural Science Foundation of China (51171141, 50701034), Program for New Century Excellent Talents in University (NCET-11-0431), Research Fund for the Doctoral Program of Higher Education of China (20120201110001), and the Fundamental Research Funds for the Central Universities.

\section{References}

[1] T. J. Rupert, D. S. Gianola, Y. Gan, and K. J. Hemker, "Experimental observations of stress-driven grain boundary migration," Science, vol. 326, no. 5960, pp. 1686-1690, 2009.

[2] M. Legros, D. S. Gianola, and K. J. Hemker, "In situ TEM observations of fast grain-boundary motion in stressed nanocrystalline aluminum films," Acta Materialia, vol. 56, no. 14, pp. 3380-3393, 2008.

[3] J. C. M. Li, "Mechanical grain growth in nanocrystalline copper," Physical Review Letters, vol. 96, no. 21, Article ID 215506, 4 pages, 2006.

[4] D. S. Gianola, S. van Petegem, M. Legros, S. Brandstetter, H. van Swygenhoven, and K. J. Hemker, "Stress-assisted discontinuous grain growth and its effect on the deformation behavior of nanocrystalline aluminum thin films," Acta Materialia, vol. 54, no. 8, pp. 2253-2263, 2006.

[5] K. Zhang, J. R. Weertman, and J. A. Eastman, "Rapid stressdriven grain coarsening in nanocrystalline $\mathrm{Cu}$ at ambient and cryogenic temperatures," Applied Physics Letters, vol. 87, no. 6, Article ID 061921, 3 pages, 2005.

[6] K. Zhang, J. R. Weertman, and J. A. Eastman, “The influence of time, temperature, and grain size on indentation creep in high-purity nanocrystalline and ultrafine grain copper," Applied Physics Letters, vol. 85, no. 22, pp. 5197-5199, 2004.

[7] M. Ames, J. Markmann, R. Karos, A. Michels, A. Tschöpe, and R. Birringer, "Unraveling the nature of room temperature grain growth in nanocrystalline materials," Acta Materialia, vol. 56, no. 16, pp. 4255-4266, 2008.

[8] X. Z. Liao, A. R. Kilmametov, R. Z. Valiev et al., "High-pressure torsion-induced grain growth in electrodeposited nanocrystalline Ni," Applied Physics Letters, vol. 88, no. 2, Article ID 021909, 3 pages, 2006.

[9] D. S. Gianola, D. H. Warner, J. F. Molinari, and K. J. Hemker, "Increased strain rate sensitivity due to stress-coupled grain growth in nanocrystalline Al," Scripta Materialia, vol. 55, no. 7, pp. 649-652, 2006.

[10] M. Dao, L. Lu, R. J. Asaro, J. T. M. de Hosson, and E. Ma, "Toward a quantitative understanding of mechanical behavior of nanocrystalline metals," Acta Materialia, vol. 55, no. 12, pp. 4041-4065, 2007. 
[11] T. Zhu and J. Li, "Ultra-strength materials," Progress in Materials Science, vol. 55, no. 7, pp. 710-757, 2010.

[12] J. A. Wert, X. Huang, G. Winther, W. Pantleon, and H. F. Poulsen, "Revealing deformation microstructures," Materials Today, vol. 10, no. 9, pp. 24-32, 2007.

[13] J. Chen, L. Lu, and K. Lu, "Hardness and strain rate sensitivity of nanocrystalline Cu," Scripta Materialia, vol. 54, no. 11, pp. 19131918, 2006.

[14] B. Zhu, R. J. Asaro, P. Krysl, K. Zhang, and J. R. Weertman, "Effects of grain size distribution on the mechanical response of nanocrystalline metals: part II," Acta Materialia, vol. 54, no. 12, pp. 3307-3320, 2006.

[15] X. Y. Li, Y. J. Wei, W. Yang, and H. J. Gao, "Competing grainboundary- and dislocation-mediated mechanisms in plastic strain recovery in nanocrystalline aluminum," Proceedings of the National Academy of Sciences of the United States of America, vol. 106, no. 38, pp. 16108-16113, 2009.

[16] J. Rajagopalan, J. H. Han, and M. T. A. Saif, "Plastic deformation recovery in freestanding nanocrystalline aluminum and gold thin films," Science, vol. 315, no. 5820, pp. 1831-1834, 2007.

[17] J. Rajagopalan, J. H. Han, and M. T. A. Saif, "On plastic strain recovery in freestanding nanocrystalline metal thin films," Scripta Materialia, vol. 59, no. 9, pp. 921-926, 2008.

[18] Y. J. Wei, A. F. Bower, and H. J. Gao, "Recoverable creep deformation due to heterogeneous grain-boundary diffusion and sliding," Scripta Materialia, vol. 57, no. 10, pp. 933-936, 2007.

[19] Z. W. Shan, R. K. Mishra, S. A. Syed Asif, O. L. Warren, and A. M. Minor, "Mechanical annealing and source-limited deformation in submicrometre-diameter Nicrystals," Nature Materials, vol. 7, no. 2, pp. 115-119, 2008.

[20] D. Jang and J. R. Greer, "Size-induced weakening and grain boundary-assisted deformation in $60 \mathrm{~nm}$ grained Ni nanopillars," Scripta Materialia, vol. 64, no. 1, pp. 77-80, 2011.

[21] S. V. Bobylev and I. A. Ovid'ko, "Grain boundary rotations in solids," Physical Review Letters, vol. 109, no. 17, Article ID 175501, 5 pages, 2012.

[22] M. Jin, A. M. Minor, E. A. Stach, and J. W. Morris Jr., "Direct observation of deformation-induced grain growth during the nanoindentation of ultrafine-grained $\mathrm{Al}$ at room temperature," Acta Materialia, vol. 52, no. 18, pp. 5381-5387, 2004.

[23] R. S. Kottada and A. H. Chokshi, "Low temperature compressive creep in electrodeposited nanocrystalline nickel," Scripta Materialia, vol. 53, no. 8, pp. 887-892, 2005.

[24] G. J. Fan, L. F. Fu, D. C. Qiao, H. Choo, P. K. Liaw, and N. D. Browning, "Grain growth in a bulk nanocrystalline Co alloy during tensile plastic deformation," Scripta Materialia, vol. 54, no. 12, pp. 2137-2141, 2006.

[25] G. J. Fan, L. F. Fu, H. Choo, P. K. Liaw, and N. D. Browning, "Uniaxial tensile plastic deformation and grain growth of bulk nanocrystalline alloys," Acta Materialia, vol. 54, no. 18, pp. 47814792, 2006.

[26] D. Pan, T. G. Nieh, and M. W. Chen, "Strengthening and softening of nanocrystalline nickel during multistep nanoindentation," Applied Physics Letters, vol. 88, no. 16, Article ID 161922, 2006.

[27] S. Brandstetter, K. Zhang, A. Escuadro, J. R. Weertman, and H. van Swygenhoven, "Grain coarsening during compression of bulk nanocrystalline nickel and copper," Scripta Materialia, vol. 58, no. 1, pp. 61-64, 2008.

[28] Y. B. Wang, B. Q. Li, M. L. Sui, and S. X. Mao, "Deformationinduced grain rotation and growth in nanocrystalline $\mathrm{Ni}$," Applied Physics Letters, vol. 92, no. 1, Article ID 011903, 2008.
[29] A. Kulovits, S. X. Mao, and J. M. K. Wiezorek, "Microstructural changes of nanocrystalline nickel during cold rolling," Acta Materialia, vol. 56, no. 17, pp. 4836-4845, 2008.

[30] B. L. Boyce and H. A. Padilla II, "Anomalous fatigue behavior and fatigue-induced grain growth in nanocrystalline nickel alloys," Metallurgical and Materials Transactions A, vol. 42, no. 7, pp. 1793-1804, 2011.

[31] Z. H. Cao, P. Y. Li, Z. H. Jiang, and X. K. Meng, "Rolling deformation induced reduction of rate sensitivity and enhancement of hardness in nanocrystalline NiFe alloys," Journal of Physics D, vol. 44, no. 29, Article ID 295403, 2011.

[32] J. A. Sharon, P.-C. Su, F. B. Prinz, and K. J. Hemker, "Stressdriven grain growth in nanocrystalline Pt thin films," Scripta Materialia, vol. 64, no. 1, pp. 25-28, 2011.

[33] S. Ni, Y. B. Wang, X. Z. Liao et al., "Grain growth and dislocation density evolution in a nanocrystalline Ni-Fe alloy induced by high-pressure torsion," Scripta Materialia, vol. 64, no. 4, pp. 327-330, 2011.

[34] H. van Swygenhoven, P. M. Derlet, and A. G. Frøseth, "Nucleation and propagation of dislocations in nanocrystalline fcc metals," Acta Materialia, vol. 54, no. 7, pp. 1975-1983, 2006.

[35] Z. Budrovic, H. van Swygenhoven, P. M. Derlet, S. van Petegem, and B. Schmitt, "Plastic deformation with reversible peak broadening in nanocrystalline nickel," Science, vol. 304, no. 5668, pp. 273-276, 2004.

[36] D. Caillard, F. Mompiou, and M. Legros, "Grain-boundary shear-migration coupling. II. Geometrical model for general boundaries," Acta Materialia, vol. 57, no. 8, pp. 2390-2402, 2009.

[37] M. Velasco, H. van Swygenhoven, and C. Brandl, "Coupled grain boundary motion in a nanocrystalline grain boundary network," Scripta Materialia, vol. 65, no. 2, pp. 151-154, 2011.

[38] J. W. Cahn and J. E. Taylor, "A unified approach to motion of grain boundaries, relative tangential translation along grain boundaries, and grain rotation," Acta Materialia, vol. 52, no. 16, pp. 4887-4898, 2004.

[39] P. L. Gai, K. Zhang, and J. Weertman, "Electron microscopy study of nanocrystalline copper deformed by a microhardness indenter," Scripta Materialia, vol. 56, no. 1, pp. 25-28, 2007. 

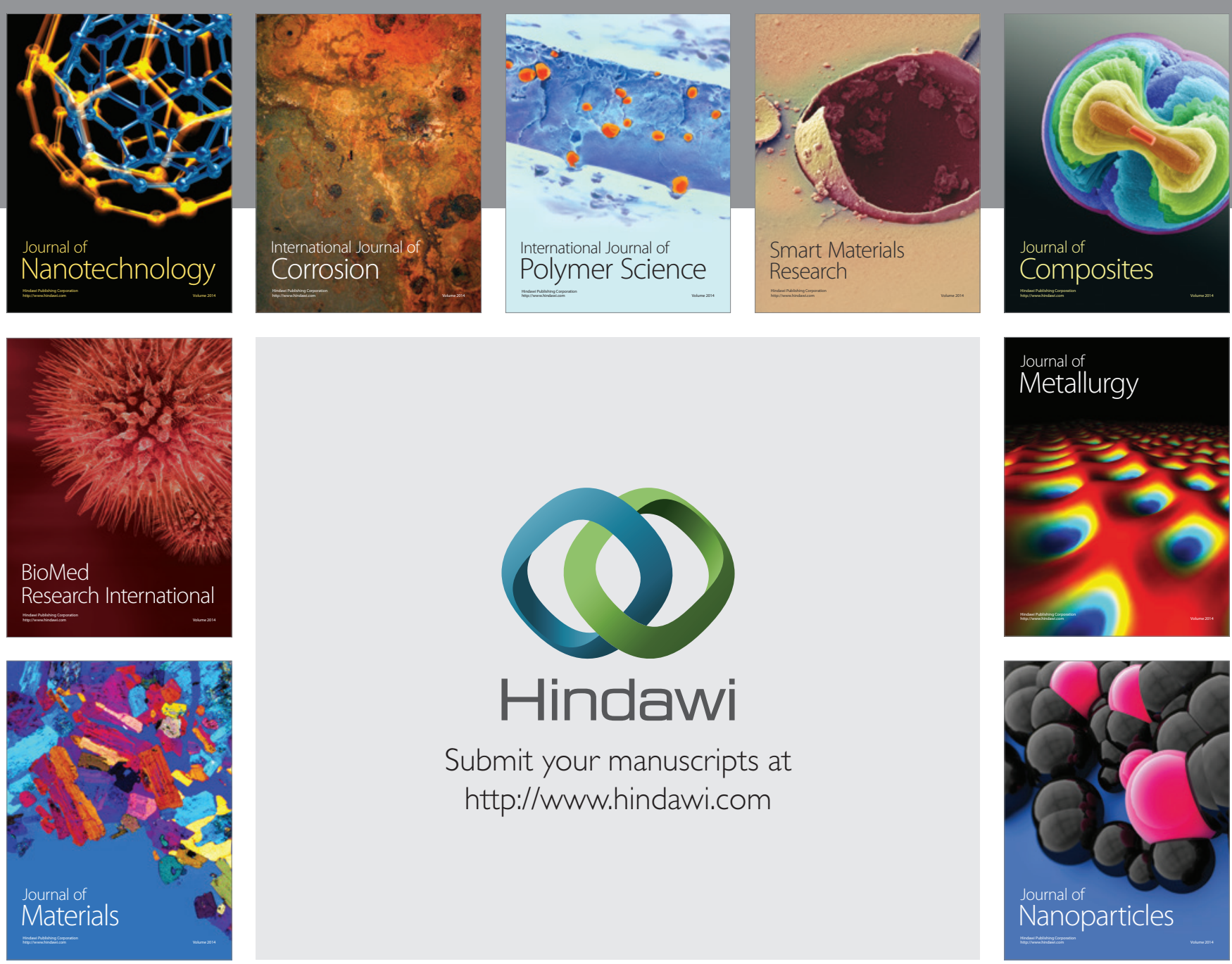

Submit your manuscripts at http://www.hindawi.com
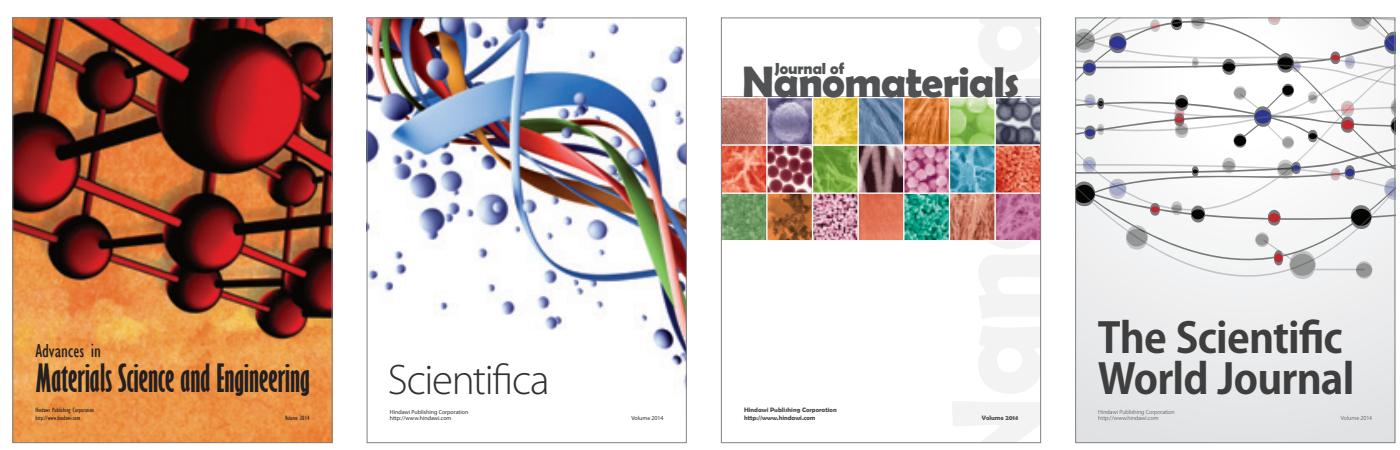

\section{The Scientific World Journal}
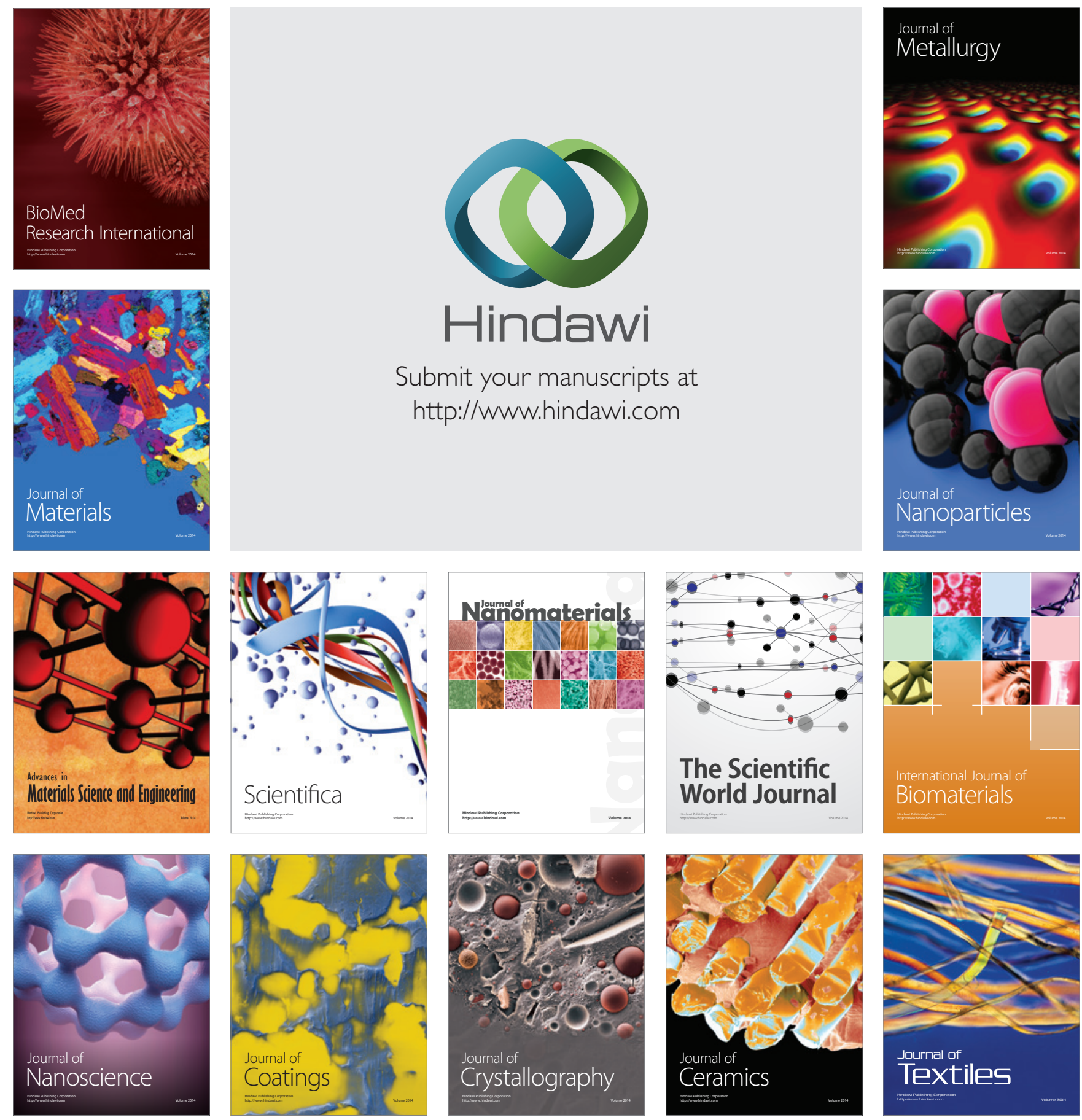